\title{
Numerical Inversion for the Multiple Fractional Orders in the Multiterm TFDE
}

\author{
Chunlong Sun, ${ }^{1,2}$ Gongsheng Li, ${ }^{1}$ and Xianzheng Jia ${ }^{1}$ \\ ${ }^{1}$ School of Science, Shandong University of Technology, Zibo 255049, China \\ ${ }^{2}$ Department of Mathematics, Southeast University, Nanjing 210096, China \\ Correspondence should be addressed to Gongsheng Li; ligs@sdut.edu.cn
}

Received 9 February 2017; Accepted 2 July 2017; Published 17 August 2017

Academic Editor: Ming Mei

Copyright (C) 2017 Chunlong Sun et al. This is an open access article distributed under the Creative Commons Attribution License, which permits unrestricted use, distribution, and reproduction in any medium, provided the original work is properly cited.

The fractional order in a fractional diffusion model is a key parameter which characterizes the anomalous diffusion behaviors. This paper deals with an inverse problem of determining the multiple fractional orders in the multiterm time-fractional diffusion equation (TFDE for short) from numerics. The homotopy regularization algorithm is applied to solve the inversion problem using the finite data at one interior point in the space domain. The inversion fractional orders with random noisy data give good approximations to the exact order demonstrating the efficiency of the inversion algorithm and numerical stability of the inversion problem.

\section{Introduction}

The partial differential equations of fractional order have played an important role in modeling of the anomalous phenomena and in the theory of the complex systems during the last two decades; see, for example, [1-8]. The so-called time-fractional diffusion equation (TFDE) that is obtained from the classical diffusion equation by replacing the firstorder time derivative by a fractional derivative of order $\alpha$ with $0<\alpha<1$ has to be especially mentioned. On the other hand, by the attempts to describe some real processes with the equations of the fractional order, several researches were confronted with the situation that the order $\alpha$ of the timefractional derivative from the corresponding models did not remain constant and changed, say, in the interval from 0 to 1 , from 1 to 2 , or even from 0 to 2 . To manage these phenomena, several approaches were suggested. One of them introduces the fractional derivatives of the variable order, that is, the derivatives with the order that can change with the time or/and depending on the spatial coordinates [9-11], and the other way is to employ the distributed order TFDE, or the multiterm TFDE in discretization. Let $\Omega$ be a bounded domain in $\mathbf{R}^{\mathbf{d}}(d \geq 1)$ with smooth boundary $\partial \Omega$, and let $T>0$; the multiterm homogeneous TFDE with variable coefficient in $\Omega$ is given as

$$
\frac{\partial^{\alpha} u}{\partial t^{\alpha}}+\sum_{s=1}^{S} r_{s} \frac{\partial^{\beta_{s}} u}{\partial t^{\beta_{s}}}=\nabla \cdot(D \nabla u), \quad x \in \Omega, 0<t \leq T,
$$

where $u=u(x, t)$ denotes the state variable at space point $x$ and time $t, \alpha$ denotes the principal fractional order, and $\beta_{1}, \beta_{2}, \ldots, \beta_{S}$ are the multiterm fractional orders of the time derivatives, which satisfy the condition

$$
0<\beta_{S}<\beta_{S-1}<\cdots<\beta_{1}<\alpha<1,
$$

and $r_{1}, r_{2}, \ldots, r_{S}$ are positive constants, and $D(x)>0$ is the smooth diffusion coefficient tensor. All of the above timefractional derivatives are defined in the sense of Caputo; for example, the fractional derivative of the order $\beta \in(0,1)$ is given by

$$
\frac{\partial^{\beta} u}{\partial t^{\beta}}=\frac{1}{\Gamma(1-\beta)} \int_{0}^{t} \frac{\partial u(x, s)}{\partial s} \frac{d s}{(t-s)^{\beta}} .
$$

See, for example, Podlubny [12] and Kilbas et al. [13] for the definition and properties of Caputo's derivative.

There are still a few research works reported on the multiterm TFDE like (1). On theoretical analysis and analytical methods for the forward problem, we refer to DaftardarGejji and Bhalekar [14], Luchko [15, 16], Jiang et al. [17], 
Ding et al. [18, 19], and $\mathrm{Li}$ et al. [20], and for numerical methods and simulations we refer to [21-23], and so on.

However, for real problems, the fractional orders, the initial distribution, the diffusion coefficient, or the source term cannot be obtained directly and we have to determine them by some additional measurements, which contributes to inverse problems arising in the fractional diffusion models. There are still some researches on inverse problems for the one-term TFDE; see, for example, Murio [24], Liu et al. [25, 26], Sakamoto and Yamamoto [27], Tuan [28], Chi et al. [29], Yamamoto and Zhang [30], Luchko et al. [31], Wei et al. $[32,33]$, and Liu et al. [34]; also see Jin and Rundell [35] for a tutorial review on inverse problems for anomalous diffusion processes.

It is noted that the research works stated above are almost related to coefficient identification problems in the one-term time/space fractional diffusion equations. However, it is also important to deal with inverse problems of determining the fractional orders in the fractional differential equations since the fractional order is an essential index characterizing the anomalous diffusion. As for inverse problems of determining fractional orders in the single-term time/space fractional diffusion models, we refer to [36-42], and so on. On the other hand, there are few literatures concerned with the inverse problems in the multiterm TFDEs to our knowledge. Li and Yamamoto [39] studied an inverse problem of identifying the multiple fractional orders in the multiterm TFDE, and they gave the uniqueness result using Laplace transform and analytical method, and later they considered the similar model [42], and also the uniqueness of determining the fractional orders, the number of the fractional terms, and the spatially varying coefficient simultaneously is proved. Recently, Sun et al. [43] considered a simultaneous inversion problem for determining the space-dependent diffusion and source coefficients in the multiterm TFDE using the optimal perturbation regularization algorithm, and quite a few numerical inversions are presented.

Based on the above analysis, we are to deal with the inverse problem of determining the multiple fractional orders in the multiterm TFDE with the additional measurements at the interior point from numerics. The uniqueness results for such kind of inverse problems have been obtained (c.f. $[39,42]$, e.g.), but numerical inversions are still open to be implemented. Based on the difference solution to the forward problem, we perform numerical inversions by utilizing the homotopy regularization algorithm not only with the accurate data but also with random noisy data. The inversion fractional orders approximate to the exact orders as the noise level gets smaller demonstrating a numerical stability of the inverse problem here.

The rest of the paper is organized as follows. In Section 2, an implicit finite difference solution to the forward problem is given and the inverse problem of determining the fractional orders is formulated. In Section 3, the homotopy regularization algorithm is introduced to solve the inversion problem and numerical inversions are presented, and concluding remarks are given in Section 4.

\section{The Forward Problem and the Inverse Problem}

Consider the forward problem given by (1) with the initial condition

$$
u(x, 0)=u_{0}(x), \quad x \in \Omega \text {, }
$$

and the homogeneous Dirichlet boundary condition

$$
\left.u\right|_{\partial \Omega}=0, \quad 0<t \leq T
$$

where the initial function is smooth enough and satisfies the consistency condition with the boundary condition. Let the diffusion coefficient $D(x)$ be $C^{1}$-class and take positive values on $\bar{\Omega}$; then the forward problem (1), (4)-(5) has a unique solution for suitable initial functions (c.f. [20], e.g.). Here we focus our attention on the finite difference solution to the forward problem. For completeness of the paper, we give an implicit finite difference scheme in $1 \mathrm{D}$ case for solving the forward problem. For further details, see [22, 23], and so on.

2.1. The Difference Scheme to the Forward Problem. Let $\Omega=$ $(0, l)$ for $l>0$. For given integer numbers $M$ and $N$, discretizing the space domain by $x_{i}=i h(i=0,1, \ldots, M)$ and the time domain by $t_{n}=n \tau(n=0,1, \ldots, N)$, we have by definition (3)

$$
\begin{aligned}
& \frac{\partial^{\alpha} u}{\partial t^{\alpha}}\left(x_{i}, t_{n+1}\right)=\frac{\tau^{-\alpha}}{\Gamma(2-\alpha)} \\
& \quad \cdot \sum_{k=0}^{n}\left[u\left(x_{i}, t_{n+1-k}\right)-u\left(x_{i}, t_{n-k}\right)\right]\left[(k+1)^{1-\alpha}-k^{1-\alpha}\right] \\
& \quad+O(\tau), \\
& \frac{\partial^{\beta_{s}} u}{\partial t^{\beta_{s}}}\left(x_{i}, t_{n+1}\right)=\frac{\tau^{-\beta_{s}}}{\Gamma\left(2-\beta_{s}\right)} \\
& \quad \cdot \sum_{k=0}^{n}\left[u\left(x_{i}, t_{n+1-k}\right)-u\left(x_{i}, t_{n-k}\right)\right]\left[(k+1)^{1-\beta_{s}}\right. \\
& \left.\quad-k^{1-\beta_{s}}\right]+O(\tau),
\end{aligned}
$$

for $s=1,2, \ldots, S$, respectively; here $h=l / M$ is the space mesh step and $\tau=T / N$ is the time mesh step.

Let $D(x) \in C^{1}(\bar{\Omega})$ and $D(x)>0$ for $x \in \bar{\Omega}$. By discretizing the term $(\partial / \partial x)\left(D(x) u_{x}\right)$ using the ordinary integer-order difference method and denoting $u_{i}^{n} \approx u\left(x_{i}, t_{n}\right)$ and $D_{i}=$ $D\left(x_{i}\right)$, we get

$$
\begin{aligned}
& \left(u_{i}^{n+1}-u_{i}^{n}\right)\left[1+\sum_{s=1}^{S} r_{i} \tau^{\alpha-\beta_{s}} \frac{\Gamma(2-\alpha)}{\Gamma\left(2-\beta_{s}\right)}\right] \\
& +\sum_{k=1}^{n}\left(u_{i}^{n+1-k}-u_{i}^{n-k}\right)\left\{\left[(k+1)^{1-\alpha}-k^{1-\alpha}\right]\right. \\
& \left.+\sum_{s=1}^{S} r_{s}\left[(k+1)^{1-\beta_{s}}-k^{1-\beta_{s}}\right] \tau^{\alpha-\beta_{s}} \frac{\Gamma(2-\alpha)}{\Gamma\left(2-\beta_{s}\right)}\right\}
\end{aligned}
$$




$$
\begin{aligned}
& =\frac{D_{i} \tau^{\alpha} \Gamma(2-\alpha)}{h^{2}}\left(u_{i-1}^{n+1}-2 u_{i}^{n+1}+u_{i+1}^{n+1}\right) \\
& +\frac{\left(D_{i}-D_{i-1}\right) \tau^{\alpha} \Gamma(2-\alpha)}{h^{2}}\left(u_{i}^{n+1}-u_{i-1}^{n+1}\right)+R^{n+1},
\end{aligned}
$$

where $R^{n}=O\left(\tau^{\alpha} h^{2}+\tau^{\alpha+1}\right)$ is the truncated term. Denoting $v=1+\sum_{s=1}^{S} r_{s} \tau^{\alpha-\beta_{s}}\left(\Gamma(2-\alpha) / \Gamma\left(2-\beta_{s}\right)\right)$, dividing by $v$ on two sides of (7), and ignoring the truncated term, we get the following:

$$
\begin{aligned}
& \left(-p_{i}+q_{i}\right) u_{i-1}^{n+1}+\left(1+2 p_{i}-q_{i}\right) u_{i}^{n+1}-p_{i} u_{i+1}^{n+1} \\
& =u_{i}^{n}-\sum_{k=1}^{n} d_{k}\left(u_{i}^{n+1-k}-u_{i}^{n-k}\right)
\end{aligned}
$$

where

$$
\begin{aligned}
p_{i}= & \frac{D_{i} \tau^{\alpha} \Gamma(2-\alpha)}{h^{2} v}, \\
q_{i}= & \frac{\left(D_{i}-D_{i-1}\right) \tau^{\alpha} \Gamma(2-\alpha)}{h^{2} v}, \\
d_{k}= & \frac{(k+1)^{1-\alpha}-k^{1-\alpha}}{v} \\
& +\sum_{s=1}^{S} \frac{(k+1)^{1-\beta_{s}}-k^{1-\beta_{s}}}{v} r_{s} \tau^{\alpha-\beta_{s}} \frac{\Gamma(2-\alpha)}{\Gamma\left(2-\beta_{s}\right)}
\end{aligned}
$$

for $k=1,2, \ldots, n$. The initial boundary value conditions are discretized as

$$
\begin{aligned}
u_{i}^{0} & =u_{0}\left(x_{i}\right) ; \\
u_{0}^{n} & =0, \\
u_{M}^{n} & =0 .
\end{aligned}
$$

Let

$$
\begin{aligned}
U^{n} & =\left(u_{1}^{n}, u_{2}^{n}, \ldots, u_{M-1}^{n}\right) ; \\
U^{0} & =\left(u_{1}^{0}, u_{2}^{0}, \ldots, u_{M-1}^{0}\right), \\
c_{k} & = \begin{cases}1-d_{1}, & k=1, \\
d_{k-1}-d_{k}, & k=2, \ldots, n ;\end{cases}
\end{aligned}
$$

and $B=\left(b_{i j}\right)_{(M-1) \times(M-1)}$, where $b_{i j}$ is defined by

$$
b_{i j}= \begin{cases}0, & j>i+1, \\ -p_{i}, & j=i+1, \\ 1+2 p_{i}-q_{i}, & j=i, \\ -\left(p_{i}-q_{i}\right), & j=i-1, \\ 0, & j<i-1,\end{cases}
$$

for $i=1,2, \ldots, M-1, j=1,2, \ldots, M-1$.
Thus, we have the implicit finite difference scheme in the matrix form given as

$$
\begin{aligned}
B U^{1} & =U^{0} \\
B U^{n+1} & =c_{1} U^{n}+c_{2} U^{n-1}+\cdots+c_{n} U^{1}+d_{n} U^{0} .
\end{aligned}
$$

Theorem 1. The implicit difference scheme (13) has only one solution, and it is of unconditional stability and convergence for any finite time $T>0$.

Proof. By the assumptions for the diffusion coefficient $D(x)$, we have $p_{i}>0$ and $p_{i}-q_{i}>0$ for $i=1, \ldots, M-1$. So the coefficient matrix $B$ of (13) is strictly diagonally dominant; then the difference equation (13) has only one solution. Moreover, thanks to the equality $\sum_{k=1}^{n} c_{k}+d_{n}=1$ and with a similar method as used in [22], we get the unconditional stability and convergence of the difference scheme for any given finite time $T>0$.

2.2. The Inverse Problem. For the forward problem (1), (4)(5), if the fractional orders $\alpha$ and $\beta_{s}(s=1, \ldots, S)$ in (1) are unknown, we encounter the inverse problem of determining these multiple fractional orders. Suppose that there are some measured points in the space domain; for example, let $x_{0} \in \Omega$ be the measured point, and we have the additional information given as

$$
u\left(x_{0}, t\right)=\theta(t), \quad 0<t \leq T,
$$

and the inverse problem is to determine $\alpha \in(0,1)$ and $\beta_{s} \epsilon$ $(0,1)(s=1, \ldots, S)$ using the overposed condition (14) based on (1) and the initial boundary conditions (4) and (5).

As stated in Section 1, the general uniqueness results have been proved in $[39,42]$ by Dr. Li and Professor Yamamoto. We give the following lemma for the completeness of the paper.

Lemma 2 (see [39]). Suppose that the fractional orders $\alpha$ and $\beta_{s}(s=1, \ldots, S)$, the diffusion coefficient tensor $D(x)$, and the coefficients $r_{s}(s=1, \ldots, S)$ in (1) satisfy the conditions given in Section 1, and the initial function $u_{0}(x)$ is smooth enough, and $u_{0}(x) \geq 0$ and $u_{0}(x) \neq \equiv$ for $x \in \Omega$. Then all the fractional orders can be determined uniquely by the additional data $\left\{u\left(x_{0}, t\right)=\theta(t)\right\}$ for $t \in(0, T)$.

The uniqueness result is very important for inverse problems in theory. By Lemma 2 we need to utilize the additional data $\theta\left(t_{i}\right)$ measured at $x_{0}$ for $i=1,2, \ldots$; however, in concrete computations, we find that the numerical inversions can also be performed only employing a few of the additional data. So it is still meaningful to study inverse problems from numerics.

\section{The Inversion Algorithm and Numerical Inversions}

In this section, we present numerical inversions for the inverse problem of (1), (4)-(5) with (14). The inversion algorithm we utilize is the homotopy regularization algorithm (see $[43,44]$, e.g.), which is a combination of the homotopy method with the optimal perturbation algorithm. We give a sketch for the inversion algorithm in the following. 
TABLE 1: The inversion results with different fractional orders for $S=1$.

\begin{tabular}{lccc}
\hline $\mathbf{a}$ & $\mathbf{a}^{\text {inv }}$ & Err & $j$ \\
\hline$(0.9,0.1)$ & $(0.900000,0.100000)$ & $1.7583 e-9$ & 30 \\
$(0.8,0.2)$ & $(0.800000,0.200000)$ & $1.2048 e-9$ & 30 \\
$(0.7,0.3)$ & $(0.700000,0.300000)$ & $1.5710 e-8$ & 30 \\
$(0.6,0.4)$ & $(0.600000,0.400000)$ & $2.0909 e-8$ & 32 \\
$(0.4,0.3)$ & $(0.400000,0.300000)$ & $5.9200 e-8$ & 34 \\
$(0.3,0.2)$ & $(0.300000,0.200000)$ & $1.9477 e-8$ & 34 \\
$(0.2,0.1)$ & $(0.200000,0.100000)$ & $1.5804 e-7$ & 33 \\
\hline
\end{tabular}

3.1. The Homotopy Regularization Algorithm. For the fractional orders $\alpha$ and $\beta_{s}(s=1,2, \ldots, S)$ satisfying the order condition $(2)$, let $I=(0,1) \times(0,1) \times \cdots \times(0,1) \subset \mathbf{R}^{S+1}$. We denote a vector

$$
\mathbf{a}=\left(\alpha, \beta_{1}, \beta_{2}, \ldots, \beta_{S}\right) \in I
$$

and equip the Euclidean norm

$$
\|\mathbf{a}\|=\sqrt{\alpha^{2}+\beta_{1}^{2}+\cdots+\beta_{S}^{2}} .
$$

For any given $\mathbf{a} \in I$, denote $u(\mathbf{a})(x, t)$ as the unique solution to the forward problem. Combined with the additional information (14), solving the inverse problem is equivalent to a minimization problem

$$
\min _{\mathbf{a} \in I}\left\|u(\mathbf{a})\left(x_{0}, t\right)-\theta(t)\right\|_{L^{2}(0, T)}^{2},
$$

where $\left\|u(\mathbf{a})\left(x_{0}, t\right)-\theta(t)\right\|_{L^{2}(0, T)}^{2}=\int_{0}^{T}\left[u(\mathbf{a})\left(x_{0}, t\right)-\theta(t)\right]^{2} d t$.

Following the homotopy regularization idea, it turns out that the following minimization problem is solved instead of (17):

$$
\min _{\mathbf{a} \in I}\left\{(1-\mu)\left\|u(\mathbf{a})\left(x_{0}, t\right)-\theta(t)\right\|_{L^{2}(0, T)}^{2}+\mu\|\mathbf{a}\|^{2}\right\},
$$

where $\mu \in(0,1)$ is the homotopy parameter which takes values from near 1 decreasingly approximating to 0 . By linearization and numerical differentiation approximations, solving (18) is transformed to solve a normal equation combined with an iteration process via

$$
\begin{aligned}
\left(\mu E+(1-\mu) \mathbf{G}^{T} \mathbf{G}\right) \delta \mathbf{a}_{j} & =(1-\mu) \mathbf{G}^{T}(\eta-\xi), \\
\mathbf{a}_{j}+\delta \mathbf{a}_{j} & \longrightarrow \mathbf{a}_{j+1}, \quad j=0,1, \ldots,
\end{aligned}
$$

where $\delta \mathbf{a}_{j}$ is a perturbation vector for any given $\mathbf{a}_{j} \in I, j$ denotes the iterative number, and $\mathbf{a}_{0}$ is the initial iteration, and

$$
\begin{aligned}
\mathbf{G} & =\left(g_{q i}\right)_{\mathrm{Q} *(S+1)}, \\
g_{q i} & =\frac{u\left(\mathbf{a}_{j}+\tau \mathbf{e}_{i}\right)\left(x_{0}, t_{q}\right)-u\left(\mathbf{a}_{j}\right)\left(x_{0}, t_{q}\right)}{\tau} ;
\end{aligned}
$$

here $\tau$ is the numerical differential step and $\mathbf{e}_{i}(i=1,2, \ldots, S+$ $1)$ is the unit basis vector in $\mathbf{R}^{S+1}$, and

$$
\begin{aligned}
& \xi=\left(u\left(\mathbf{a}_{j}\right)\left(x_{0}, t_{1}\right), \ldots, u\left(\mathbf{a}_{j}\right)\left(x_{0}, t_{\mathrm{Q}}\right)\right)^{T} ; \\
& \eta=\left(\theta\left(t_{1}\right), \ldots, \theta\left(t_{\mathrm{Q}}\right)\right)^{T} .
\end{aligned}
$$

The algorithm can be terminated as long as an optimal perturbation satisfies the condition $\left\|\overline{\mathbf{a}}_{j}\right\|_{2} \leq$ eps; here eps is the given convergent precision. Like that done in $[43,44]$, we employed the homotopy parameter by

$$
\mu=\mu(j)=\frac{1}{1+\exp \left(\sigma\left(j-j_{0}\right)\right)},
$$

where $j$ is the number of iterations, $j_{0}$ is a preestimated number, and $\sigma>0$ is an adjusted parameter.

In the following, we will take two-term case $(S=1)$ and three-term case $(S=2)$ as example to perform the inversion algorithm in $1 D$ case. We set the model parameters $l=\pi, T=$ 1 , and $D=1$ and $u_{0}(x)=\sin (x)$ if there is no specification. In addition, we choose $j_{0}=5$ and $\sigma=0.5$ in (22) to determine the homotopy parameter and utilize the grid steps $h=l / 100$ and $\tau=T / 100$ in the computation of the forward problem by the difference scheme (13).

3.2. Inversion for $S=1$. In the case of $S=1$, let $r_{1}=0.5$, and the fractional orders we have to determine are $\alpha$ and $\beta_{1}$, and $\mathbf{a}=\left(\alpha, \beta_{1}\right)$ is the exact solution of the fractional orders. By the exact fractional orders, we compute the forward problem to get the additional data at $x_{0}=l / 2$ with which we reconstruct the fractional orders. It is noticeable that the dimension of the additional data is related to the discretization of the time domain; that is, there are $u\left(l / 2, t_{i}\right)$ for $i=0,1, \ldots, N$; here $t_{i}$ is the time at which the measurement is made, and we have a $N+1$-dimensional vector representing the additional data given as

$$
\left(u\left(\frac{l}{2}, t_{0}\right), u\left(\frac{l}{2}, t_{1}\right), \ldots, u\left(\frac{l}{2}, t_{N}\right)\right) .
$$

However, the inversion could be performed using a few of the additional data, and it is meaningful to investigate the influence of the number (dimension) of the additional data on the inversion algorithm.

3.2.1. Inversion with Accurate Data. Taking two additional datasets at the time of $t=0.3$ and $t=0.4$ and choosing the initial iteration as $\mathbf{a}_{0}=\mathbf{0}$, the inversion results are listed in Table 1, where $\mathbf{a}^{\text {inv }}$ is the inversion solution, Err $=\| \mathbf{a}-$ $\mathbf{a}^{\text {inv }}\|/\| \mathbf{a} \|$ denotes the solutions error, and $j$ is the number of iterations.

Without loss of generality, choosing the exact fractional orders as $\alpha=0.8$ and $\beta_{1}=0.4$, we implement the inversion 
TABLE 2: The inversion results with number of the additional data for $S=1$.

\begin{tabular}{|c|c|c|c|c|}
\hline$L$ & {$[t]$} & $a^{\text {inv }}$ & Err & $j$ \\
\hline 2 & {$[0.3,0.4]$} & $(0.800000,0.400000)$ & $2.569 e-8$ & 30 \\
\hline 3 & {$[0.3,0.4,0.5]$} & $(0.800000,0.400000)$ & $3.288 e-9$ & 28 \\
\hline 4 & {$[0.3,0.4,0.5,0.6]$} & $(0.800000,0.400000)$ & $1.042 e-8$ & 26 \\
\hline 5 & {$[0.3,0.4,0.5,0.6,0.7]$} & $(0.800000,0.400000)$ & $3.712 e-9$ & 25 \\
\hline 6 & {$[0.3,0.4,0.5,0.6,0.7,0.8]$} & $(0.800000,0.400000)$ & $3.448 e-9$ & 24 \\
\hline 7 & {$[0.2,0.3,0.4,0.5,0.6,0.7,0.8]$} & $(0.800000,0.400000)$ & $6.181 e-9$ & 23 \\
\hline 8 & {$[0.2,0.3,0.4,0.5,0.6,0.7,0.8,0.9]$} & $(0.800000,0.400000)$ & $1.885 e-8$ & 22 \\
\hline 9 & {$[0.1,0.2,0.3,0.4,0.5,0.6,0.7,0.8,0.9]$} & $(0.800000,0.400000)$ & $9.076 e-9$ & 22 \\
\hline
\end{tabular}

TABLE 3: The inversion results using noisy data for $S=1$.

\begin{tabular}{lccc}
\hline$\varepsilon$ & $\overline{\mathbf{a}}^{\text {inv }}$ & $\bar{E}$ rr & $\bar{j}$ \\
\hline $5 \%$ & $(0.789840,0.422128)$ & $2.7223 e-2$ & 14.6 \\
$1 \%$ & $(0.799757,0.401140)$ & $1.3032 e-3$ & 14.3 \\
$0.1 \%$ & $(0.799990,0.400094)$ & $1.0588 e-4$ & 13.9 \\
$0.01 \%$ & $(0.799999,0.400009)$ & $1.0086 e-5$ & 15.0 \\
$0.001 \%$ & $(0.800000,0.400001)$ & $9.6878 e-7$ & 16.0 \\
\hline
\end{tabular}

algorithm using different number of the additional data. The inversion results are listed in Table 2 , where $L$ denotes the number (dimension) of the additional data and $[t]$ denotes the measured time to get the additional data.

From Table 1, it can be seen that the inversion can be realized only utilizing two additional datasets, and the inversion solutions are good approximations to the exact solutions. From Table 2, we can see that there are some influences for choosing the number of the additional data on the inversion algorithm, and the inversion results become a little better as the number of the additional data increases and the number of the iterations decreases. Moreover, if using much more additional data in the above, for example, taking all of the measured data given by (23), the inversion solution is still $\mathbf{a}^{\text {inv }}=(0.800000,0.400000)$, and the solutions error is Err $=2.4636 e-8$, but the number of iterations decreases to $j=17$.

3.2.2. Inversion with Noisy Data. It is difficult to perform an inversion algorithm in the case of using random noisy data, especially for inverse problems arising from the fractional diffusion. Noting computational errors and data noises, the additional information utilized for real inversions is often given as

$$
\theta^{\varepsilon}(t)=\theta(t)+\varepsilon \zeta
$$

where $\varepsilon>0$ is the noise level and $\zeta$ is a random vector ranging within $[-1,1]$.

Also we take the exact fractional orders as $\alpha=0.8$ and $\beta=0.4$; that is, the exact solution of the inverse problem here is given as $\mathbf{a}=(0.8,0.4)$. It is noticeable that the inversion with data noises could always fail if still using the above inversion parameters, and we perform the inversion using the completely additional data at $t_{i}$ for $i=0,1, \ldots, N$ and choosing the initial iteration as nonzero vector of $\mathbf{a}_{\mathbf{0}}=$
$(0.1,0.1)$. The average inversion results with continuous tentime inversions are listed in Table 3 , where $\overline{\mathbf{a}}^{\text {inv }}$ is the average inversion solution of the ten-time inversions and $\bar{E} \mathrm{rr}=\left\|\overline{\mathbf{a}}^{\mathrm{inv}}-\mathbf{a}\right\|_{2} /\|\mathbf{a}\|_{2}$ is the relative average error in the solutions.

From Table 3, we find that the inversion is satisfactory in the case of using random noisy data, and the inversion errors become small when reducing the noise level.

3.3. Inversion for $S=2$. In the case of $S=2$, the fractional orders we are to determine are $\alpha, \beta_{1}$ and $\beta_{2}$, and $\mathbf{a}=$ $\left(\alpha, \beta_{1}, \beta_{2}\right)$ is the exact solution in this case. Let $r_{1}=1, r_{2}=$ 0.2 , and also choose $x_{0}=l / 2$ as the measured point. We utilize nine additional datasets at the time of $t_{i}=i / 10$ for $i=1,2, \ldots, 9$ and choose the initial iteration as $\mathbf{a}_{0}=$ $(0.1,0.05,0.01)$ here. The inversion results with accurate data are listed in Table 4.

As done for $S=1$, we can also realize the inversion in the case of using noisy data for $S=2$ although the illposedness of the inversion becomes more serious than that of $S=1$. Taking $\mathbf{a}=(0.9,0.7,0.5)$ as an example and using $\mathbf{a}_{0}=(0.3,0.2,0.1)$ as the initial iteration, the average results also with continuous ten-time inversions are listed in Table 5, where $\varepsilon, \overline{\mathbf{a}}^{\mathrm{inv}}$, and $\bar{E}$ rr are all the same as those used in Table 3. It is important to note that we perform the inversion algorithm with convergent precision as eps $=1 e-2$ for $\varepsilon=1 \%$ and $\varepsilon=0.1 \%$ and eps $=1 e-4$ for $\varepsilon=0.01 \%$ and $\varepsilon=0.001 \%$, respectively.

From Tables 3 and 5, we can see that the inversion solutions with random noisy data approximate to the exact solutions as the noise level gets smaller. Although the inversion results for $S=2$ with large noises are not so good as that for $S=1$, they are still satisfactory and show a numerical stability for the inverse problem. 
TABLE 4: The inversion results with different fractional orders for $S=2$.

\begin{tabular}{lccc}
\hline $\mathbf{a}$ & $\mathbf{a}^{\text {inv }}$ & Err & $j$ \\
\hline$(0.9,0.7,0.5)$ & $(0.900000,0.700000,0.500000)$ & $1.0216 e-9$ & 41 \\
$(0.8,0.6,0.4)$ & $(0.800000,0.600000,0.400000)$ & $4.5193 e-9$ & 40 \\
$(0.7,0.5,0.3)$ & $(0.700000,0.500000,0.300000)$ & $4.3115 e-8$ & 39 \\
$(0.6,0.5,0.4)$ & $(0.600000,0.500000,0.400000)$ & $2.9329 e-9$ & 45 \\
$(0.5,0.4,0.3)$ & $(0.500000,0.400000,0.300000)$ & $2.4011 e-9$ & 45 \\
$(0.4,0.3,0.2)$ & $(0.400000,0.300000,0.200000)$ & $8.8878 e-9$ & 45 \\
$(0.3,0.2,0.1)$ & $(0.300000,0.200000,0.100000)$ & $9.9418 e-8$ & 45 \\
\hline
\end{tabular}

TABLE 5: The inversion results using noisy data for $S=2$.

\begin{tabular}{lcc}
\hline$\varepsilon$ & $\overline{\mathbf{a}}^{\text {inv }}$ & $\bar{E}$ rr \\
\hline $1 \%$ & $(0.853161,0.762050,0.411797)$ & $9.4438 e-2$ \\
$0.1 \%$ & $(0.878925,0.716188,0.520835)$ & $2.7124 e-2$ \\
$0.01 \%$ & $(0.900086,0.699732,0.500985)$ & $8.2268 e-4$ \\
$0.001 \%$ & $(0.900007,0.699989,0.500042)$ & $3.4895 e-5$ \\
\hline
\end{tabular}

\section{Conclusions}

The numerical determination problem for the fractional orders in the multiterm TFDE is investigated using some measurements at the interior point of the space domain. The inversion problem is unique, and numerical inversions with random noisy data are performed successfully by using the homotopy regularization algorithm. The homotopy regularization algorithm can also be utilized to determine the multiple fractional orders in the multidimensional case as long as a solution to the forward problem can be worked out.

It is noted that numerical inversions can be implemented smoothly in the case that the fractional orders satisfy the order condition (2) given in Section 1, which is just a necessary condition for the uniqueness of the inverse problem. The inversion results are very satisfactory if coping with accurate data; however, they become a little bad in the case of $S=2$ when using noisy data with noises greater than $1 \%$. Therefore, we can say that the stability of the inverse problem here with more fractional derivatives, that is, $S \geq 2$, could be severely ill-posed in spite of the fact that uniqueness is valid. We will deal with the inverse problem in the multidimensional case and we have to seek more effective inversion algorithms for the multiterm TFDE with $S>2$ in the future work.

\section{Conflicts of Interest}

The authors declare that there are no conflicts of interest regarding the publication of this paper.

\section{Acknowledgments}

This work is supported by the National Natural Science Foundation of China (nos. 11371231, 11071148).

\section{References}

[1] E. E. Adams and L. W. Gelhar, "Field study of dispersion in a heterogeneous aquifer: 2. Spatial moments analysis," Water Resources Research, vol. 28, no. 12, pp. 3293-3307, 1992.
[2] D. A. Benson, The Fractional Advection-Dispersion Equation: Development and Application. Dissertation of Doctorial Degree [Dissertation, thesis], University of Nevada, Reno, Reno. USA, 1998.

[3] B. Berkowitz, H. Scher, and S. E. Silliman, "Anomalous transport in laboratory-scale, heterogeneous porous media," Water Resources Research, vol. 36, no. 1, pp. 149-158, 2000.

[4] R. Caponetto, G. Dongola, L. Fortuna, and I. Petras, Fractional Order Systems: Modeling and Control Applications, World Scientific, Singapore, 2010.

[5] M. Giona, S. Cerbelli, and H. E. Roman, "Fractional diffusion equation and relaxation in complex viscoelastic materials," Physica A: Statistical Mechanics and Its Applications, vol. 191, no. 1-4, pp. 449-453, 1992.

[6] Y. Hatano and N. Hatano, "Dispersive transport of ions in column experiments: An explanation of long-tailed profiles," Water Resources Research, vol. 34, no. 5, pp. 1027-1033, 1998.

[7] F. Mainardi, Fractional Calculus and Waves in Linear Viscoelasticity: An Introduction to Mathematical Models, Imperial College Press, London, UK, 2010.

[8] L. Zhou and H. M. Selim, "Application of the fractional advection-dispersion equation in porous media," Soil Science Society of America Journal, vol. 67, no. 4, pp. 1079-1084, 2003.

[9] C. F. Coimbra, "Mechanics with variable-order differential operators," Annalen der Physik, vol. 12, no. 11-12, pp. 692-703, 2003.

[10] C. F. Lorenzo and T. T. Hartley, "Variable order and distributed order fractional operators," Nonlinear Dynamics, vol. 29, no. 1-4, pp. 57-98, 2002.

[11] H. T. Pedro, M. H. Kobayashi, J. M. Pereira, and C. F. Coimbra, "Variable order modeling of diffusive-convective effects on the oscillatory flow past a sphere," Journal of Vibration and Control, vol. 14, no. 9-10, pp. 1659-1672, 2008.

[12] I. Podlubny, Fractional Differential Equations, vol. 198, Academic Press, San Diego, Calif, USA, 1999.

[13] A. A. Kilbas, H. M. Srivastava, and J. J. Trujillo, Theory and Applications of Fractional Differential Equations, New York, NY, USA, Elsevier, 2006.

[14] V. Daftardar-Gejji and S. Bhalekar, "Boundary value problems for multi-term fractional differential equations," Journal of 
Mathematical Analysis and Applications, vol. 345, no. 2, pp. 754$765,2008$.

[15] Y. Luchko, "Maximum principle for the generalized timefractional diffusion equation," Journal of Mathematical Analysis and Applications, vol. 351, no. 1, pp. 218-223, 2009.

[16] Y. Luchko, "Initial-boundary problems for the generalized multi-term time-fractional diffusion equation," Journal of Mathematical Analysis and Applications, vol. 374, no. 2, pp. 538$548,2011$.

[17] H. Jiang, F. Liu, I. Turner, and K. Burrage, "Analytical solutions for the multi-term time-space Caputo-Riesz fractional advection-diffusion equations on a finite domain," Journal of Mathematical Analysis and Applications, vol. 389, no. 2, pp. 11171127, 2012.

[18] X.-L. Ding and Y.-L. Jiang, "Analytical solutions for the multiterm time-space fractional advection-diffusion equations with mixed boundary conditions," Nonlinear Analysis. Real World Applications. An International Multidisciplinary Journal, vol. 14, no. 2, pp. 1026-1033, 2013.

[19] X.-L. Ding and J. J. Nieto, "Analytical solutions for the multiterm time-space fractional reaction-diffusion equations on an infinite domain," Fractional Calculus and Applied Analysis, vol. 18, no. 3, pp. 697-716, 2015.

[20] Z. Li, Y. Liu, and M. Yamamoto, "Initial-boundary value problems for multi-term time-fractional diffusion equations with positive constant coefficients," Applied Mathematics and Computation, vol. 257, pp. 381-397, 2015.

[21] B. Jin, R. Lazarov, Y. Liu, and Z. Zhou, "The Galerkin finite element method for a multi-term time-fractional diffusion equation," Journal of Computational Physics, vol. 281, pp. 825$843,2015$.

[22] G. Li, C. Sun, X. Jia, and D. Du, "Numerical solution to the multi-term time fractional diffusion equation in a finite domain," Numerical Mathematics. Theory, Methods and Applications, vol. 9, no. 3, pp. 337-357, 2016.

[23] F. Liu, M. M. Meerschaert, R. J. McGough, P. Zhuang, and Q. Liu, "Numerical methods for solving the multi-term timefractional wave-diffusion equation," Fractional Calculus and Applied Analysis, vol. 16, no. 1, pp. 9-25, 2013.

[24] D. A. Murio, "Stable numerical solution of a fractional-diffusion inverse heat conduction problem," Computers \& Mathematics with Applications, vol. 53, no. 10, pp. 1492-1501, 2007.

[25] J. J. Liu and M. Yamamoto, "A backward problem for the time-fractional diffusion equation," Applicable Analysis. An International Journal, vol. 89, no. 11, pp. 1769-1788, 2010.

[26] J. J. Liu, M. Yamamoto, and L. L. Yan, "On the reconstruction of unknown time-dependent boundary sources for time fractional diffusion process by distributing measurement," Inverse Problems, vol. 32, no. 1, article 015009, Article ID 015009, 2016.

[27] K. Sakamoto and M. Yamamoto, "Initial value/boundary value problems for fractional diffusion-wave equations and applications to some inverse problems," Journal of Mathematical Analysis and Applications, vol. 382, no. 1, pp. 426-447, 2011.

[28] V. K. Tuan, "Inverse problem for fractional diffusion equation," Fractional Calculus and Applied Analysis. An International Journal for Theory and Applications, vol. 14, no. 1, pp. 31-55, 2011.

[29] G. Chi, G. Li, and X. Jia, "Numerical inversions of a source term in the FADE with a Dirichlet boundary condition using final observations," Computers \& Mathematics with Applications, vol. 62, no. 4, pp. 1619-1626, 2011.
[30] M. Yamamoto and Y. Zhang, "Conditional stability in determining a zeroth-order coefficient in a half-order fractional diffusion equation by a Carleman estimate," Inverse Problems, vol. 28, no. 10, article 105010, 2012.

[31] Y. Luchko, W. Rundell, M. Yamamoto, and L. Zuo, "Uniqueness and reconstruction of an unknown semilinear term in a timefractional reaction-diffusion equation," Inverse Problems, vol. 29, no. 6, article 065019, Article ID 065019, 2013.

[32] T. Wei and J. Wang, "A modified quasi-boundary value method for an inverse source problem of the time-fractional diffusion equation," Applied Numerical Mathematics, vol. 78, pp. 95-111, 2014.

[33] T. Wei, X. L. Li, and Y. S. Li, "An inverse time-dependent source problem for a time-fractional diffusion equation," Inverse Problems, vol. 32, no. 8, article 085003, Article ID 085003, 2016.

[34] Y. Liu, W. Rundell, and M. Yamamoto, "Strong maximum principle for fractional diffusion equations and an application to an inverse source problem," Fractional Calculus and Applied Analysis, vol. 19, no. 4, pp. 888-906, 2016.

[35] B. Jin and W. Rundell, "A tutorial on inverse problems for anomalous diffusion processes," Inverse Problems, vol. 31, no. 3, article 035003, 2015.

[36] A. N. Bondarenko and D. S. Ivaschenko, "Numerical methods for solving inverse problems for time fractional diffusion equation with variable coefficient," Journal of Inverse and IllPosed Problems, vol. 17, no. 5, pp. 419-440, 2009.

[37] J. Cheng, J. Nakagawa, M. Yamamoto, and T. Yamazaki, "Uniqueness in an inverse problem for a one-dimensional fractional diffusion equation," Inverse Problems. An International Journal on the Theory and Practice of Inverse Problems, Inverse Methods and Computerized Inversion of Data, vol. 25, article 115002, 2009.

[38] G. Li, D. Zhang, X. Jia, and M. Yamamoto, "Simultaneous inversion for the space-dependent diffusion coefficient and the fractional order in the time-fractional diffusion equation," Inverse Problems, vol. 29, no. 6, article 065014, 2013.

[39] Z. Li and M. Yamamoto, "Uniqueness for inverse problems of determining orders of multi-term time-fractional derivatives of diffusion equation," Applicable Analysis, vol. 94, no. 3, pp. 570579,2015

[40] S. Tatar and S. Ulusoy, "A uniqueness result for an inverse problem in a space-time fractional diffusion equation," Electronic Journal of Differential Equations, vol. 2013, no. 258, pp. 1-9, 2013.

[41] S. Chen, F. Liu, X. Jiang, I. Turner, and K. Burrage, "Fast finite difference approximation for identifying parameters in a two-dimensional space-fractional nonlocal model with variable diffusivity coefficients," SIAM Journal on Numerical Analysis, vol. 54, no. 2, pp. 606-624, 2016.

[42] Z. Li, O. Y. Imanuvilov, and M. Yamamoto, "Uniqueness in inverse boundary value problems for fractional diffusion equations," Inverse Problems, vol. 32, no. 1, article 015004, 2016.

[43] C. Sun, G. Li, and X. Jia, "Simultaneous inversion for the diffusion and source coefficients in the multi-term TFDE," Inverse Problems in Science and Engineering, pp. 1-21, 2017.

[44] D. Zhang, G. Li, X. Jia, and H. Li, "Simultaneous inversion for space-dependent diffusion coefficient and source magnitude in the time fractional diffusion equation," Journal of Mathematics Research, vol. 5, no. 2, pp. 65-78, 2013. 


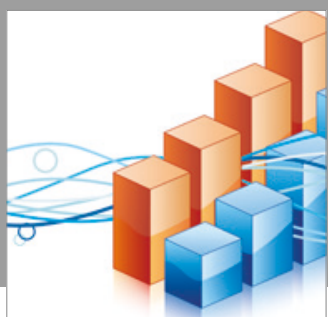

Advances in

Operations Research

vatersals

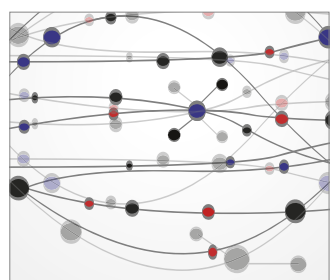

\section{The Scientific} World Journal
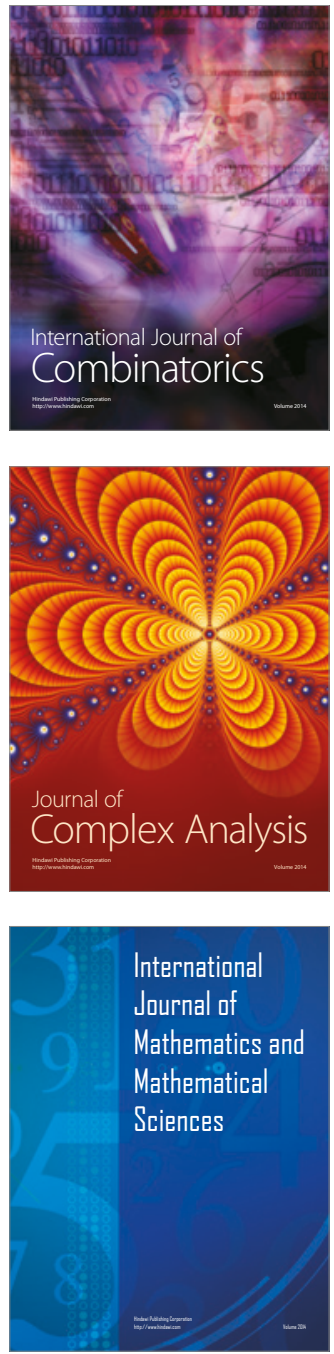
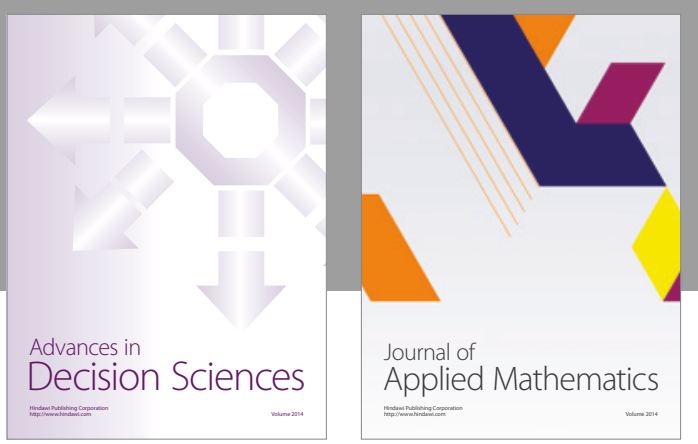

Algebra

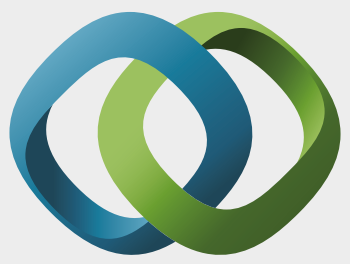

\section{Hindawi}

Submit your manuscripts at

https://www.hindawi.com
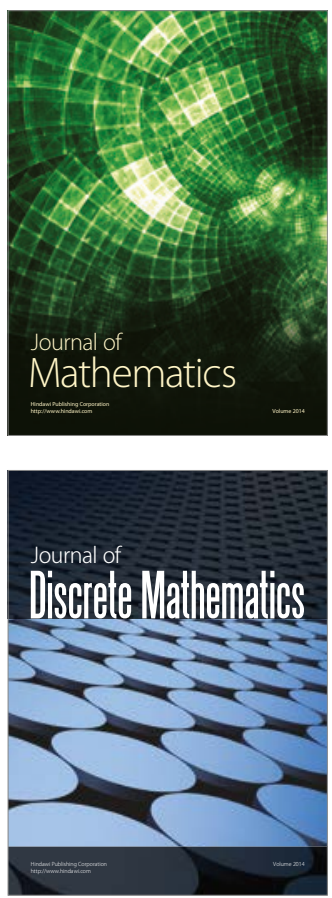

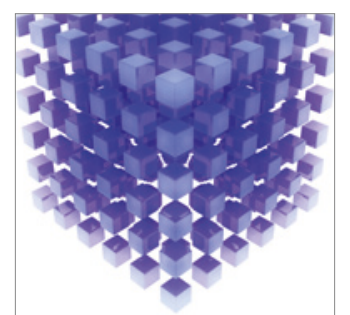

Mathematical Problems in Engineering
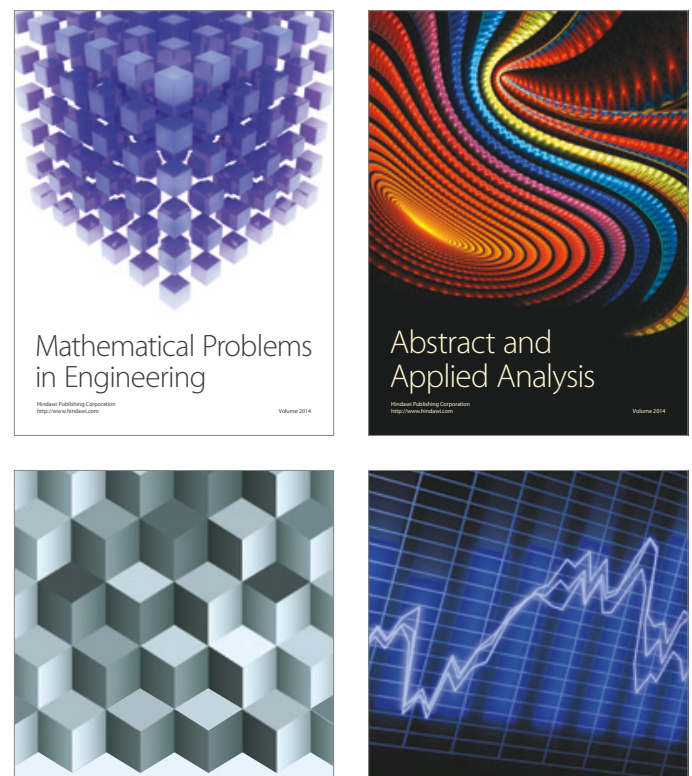

Journal of

Function Spaces

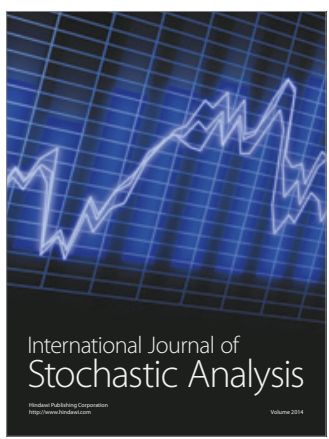

Probability and Statistics
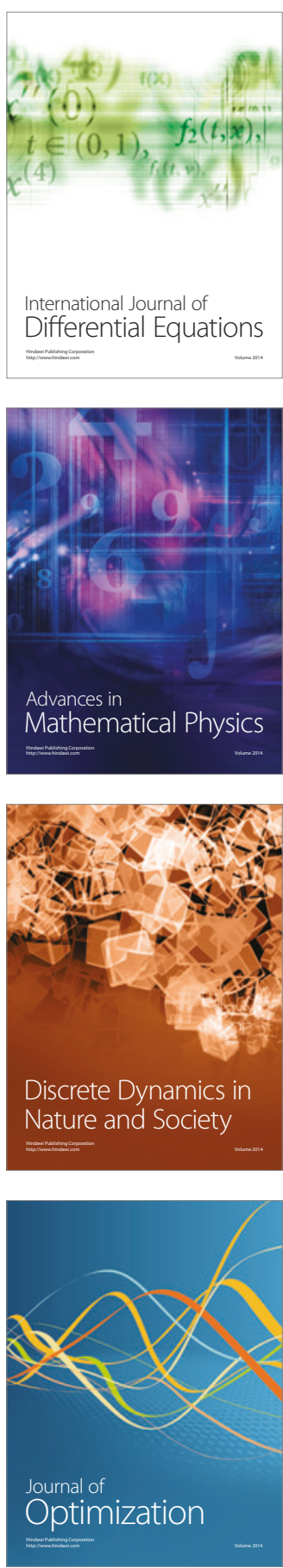